\title{
End Diastolic Volume Imaging
}

National Cancer Institute

\section{Source}

National Cancer Institute. End Diastolic Volume Imaging. NCI Thesaurus. Code C94893.

Imaged ventricular blood pool capacity within the endocardial surface in a gated scan during the time of diastole. 